- Demonstrate the patient's right to have a say in their care. (NICE, 2004).

- Demonstrate compliance with the End of Life Care Strategy (DoH, 2008).

- Legal imperative for all organisations providing NHS funded services. (National Health Services Act 2006, Local Government and Public Involvement in Health Act 2007, Equity and Excellence: Liberating the NHS, 2008)

Materials and methods A steering group was set up incorporating senior members of staff including a Trustee and the Chief Executive. This group identified various methods for involving our stakeholders which included

Interviewing patients, carers, volunteers and paid staff

Listening exercise with carers

Questionnaires Productive Ward Series

a template for writing reports on these activities

a leaflet to hand-out giving an explanation of "Involvement"

Results As a result of this we are now:-

- Piloting Nurse Led Clinics

- Opening Day Care to bereaved carers

- Using a new discharge planning template on the inpatient unit

- Implementing a "Carer's Break" scheme via Day Care

- Developing a complementary therapy strategy based on user feedback about the value of this service to them

Conclusions The moving from "meeting" to "happening" has helped to promote the principles of equality, fairness and inclusiveness by actively seeking to engage with our stakeholders including our staff.

The identification of services valued by patients and their carers has ensured that our finances are spent in addressing the needs of our patients rather than what we consider their needs to be.

\section{P153 SERVICE USER GROUP (SUG) - FROM INCEPTION TO BEYOND}

Doreen Thompson. Willen Hospice, Milton Keynes, United Kingdom

10.1136/bmjspcare-2013-000591.175

Background To establish a SUG giving patients, carers, staff and members of the public opportunity to engage in improving hospice services.

Involvement covers a range of activities, from consulting service users about their views in partnership, to aid the development of projects or services.

Ensures the views of patients, carers and the public are listened to, ensuring that we continue to maintain excellence within our service now and into the future.

Aims and objectives

- SUG established to enable the hospice to provide evidence of consultation on matters directly related to patient care.

- SUG involved in consultation on available information for service users.

- Review and approve production of literature to ensure service users receive relevant information.

- SUG involved in fund raising and promotion of hospice services.

- SUG ensures service users input into service provision, promoting standards of excellence in patient care
Approach used

- IPU Sister identified as project lead.

- Advertised to promote development of Service User Group. Invited participants to join via appropriate communication channels.

- Guest speaker utilised to drive engagement.

Outcomes The SUG is involved in decision making on hospice improvements and have participated in workshops with a local Cancer Network Group. A service user and the Network group leader were key speakers at the group conference. The SUG have worked on patient feedback and audit tools and information leaflets for patients and families.

The SUG were runners-up in the Patient Experience Network (P. E. N.) awards 2012

SUG has forged links with the local Hospital patient partnership forum. Representatives attend the meetings of both groups.

With a diverse, growing local population the SUG helps to reflect our local community needs. Their unique input helps the organisation see new perspectives on service development.

\section{P154 FOOD AND NUTRITION IN A HOSPICE: FROM AUDIT TO ACTION}

${ }^{1,2}$ Eleni Tsiompanou, ${ }^{1}$ Teresa Day, ${ }^{1}$ Andrew Knight. ${ }^{1}$ Princess Alice Hospice, Esher, UK, ${ }^{2}$ Kingston Hospital, Kingston, UK

\subsection{6/bmjspcare-2013-000591.176}

In 2011, Princess Alice Hospice (PAH) participated in the test phase of the Nutrition and Hydration Audit tool which was created jointly by the Help the Hospices Food and Nutrition Group (FNG) and the National Audit Tools Group (NATG)1. Since this pilot audit was completed a number of steps were taken to reach standards that were highlighted by the audit:

1. A 'Nutrition Requirements Tool' was introduced on the ward. Further work has been undertaken to develop this tool.

2. Understanding the patient experience is at the heart of hospice care. We asked patients on the ward and in Day Hospice to tell us what they thought of our food service. Patients told us that the food and drink service that we provide is excellent. Areas of potential improvement are: protected mealtimes; attending to cultural \& religious requirements, allergies and intolerances, special diets; giving written information re: food service; menus and food portions.

3. We provided regular teaching/training sessions for internal and external staff and volunteers. Our training enables healthcare professionals to become more involved in establishing nutrition as part of the patient's care plan and actively listen to patients and carers, offering support for their needs in relation to foods and lifestyle.

4. An eight-week support and education programme for patients and carers was designed in Day Hospice, offering up-to-date information from our multi-disciplinary team on nutrition and diet, lifestyle and symptom control.

5. Our Nutrition Steering Group meets to promote the role of the multidisciplinary approach to nutritional management and care of our patients.

Princess Alice Hospice is committed to delivering safe and high quality nutritional care to people with life-limiting illnesses, 